\title{
High-flux mitochondrial cholesterol trafficking, a specialized function of the adrenal cortex
}

\author{
Colin Jefcoate \\ Department of Pharmacology, University of Wisconsin Medical School, Madison, Wisconsin, USA \\ J. Clin. Invest. 110:881-890 (2002). doi:10.1172/JCI200216771.
}

The adrenal cortex is a tissue of excess in terms of both cholesterol metabolism and cholesterol exchange with the circulation. Exceptionally high levels of lipoprotein receptors in this highly vascularized tissue provide ready access to dietary cholesterol, allowing the adrenocortical cells to maintain impressive stores of cytoplasmic cholesterol ester (CE) droplets. Tightly packed among the CE droplets are specialized mitochondria, carrying in their inner membranes high levels of the cytochrome P450scc (CYP11A1).

This enzyme carries out the so-called side chain cleavage reaction, consuming cholesterol to produce pregnenolone, the precursor of cortisol and all other steroids. Glucocorticoid synthesis is tightly regulated at the level of cholesterol metabolism, which responds to ACTH stimulation over a period of minutes and ceases equally quickly when this hormone is removed. Remarkably, this dynamic process is modulated under most circumstances not by control of the intrinsic enzymatic activity of $\mathrm{P} 450 \mathrm{scc}$, but rather by substrate availability. For this reason, cholesterol transport within the mitochondrion has emerged as the key control point for steroidogenesis.

The adrenal cortex is not alone in requiring efficient and controlled delivery of cholesterol into mitochondria. Other steroidogenic cells, including several cell types in the ovary, the Leydig cells of the testis, and a subset of hippocampal neurons (1), also employ $\mathrm{P} 450 \mathrm{scc}$ to produce pregnenolone and a variety of downstream steroid hormones or neurosteroids. In vertebrates ranging from birds and fish (2) to mammals, these various cell types all express a short-lived mito-

\footnotetext{
Address correspondence to: Colin Jefcoate, Department of Pharmacology, University of Wisconsin Medical School,

1300 University Avenue, Madison, Wisconsin 53706, USA.

Phone: (608) 263-3975; Fax: (608) 262-1257;

E-mail: jefcoate@facstaff.wisc.edu.

Conflict of interest: No conflict of interest has been declared. Nonstandard abbreviations used: cholesterol ester (CE); steroidogenic acute regulatory protein (StAR); congenital adrenal hyperplasia (CAH); outer mitochondrial membrane (OMM); inner mitochondrial membrane (IMM); phosphorylated p30 StAR (pp30); cycloheximide (CHX); protein kinase A (PKA); peripheral benzodiazepine receptor (PBR); mitochondrial thioesterase (MTE); arachidonic acid (AA); mitogen-activated protein kinase (MAPK).
}

chondrial import factor now called the steroidogenic acute regulatory protein (StAR), which mediates this process. Here, I examine the often confusing literature on StAR's mechanism of action, particularly in light of recent work establishing the importance of other players, and I present a model for StAR's interaction with cholesterol and with some of these other proteins. I also discuss the insights into mitochondrial function that have come from the analysis of patients with congenital adrenal hyperplasia (CAH), who lack this factor. Finally, I consider the multi-tiered regulation of StAR and related proteins in adrenocortical cells and other steroidogenic cell types.

\section{A StAR is born}

Cells of the glomerulosa, fasciculata, and reticularis zones of the cortex express distinct cholesterol-processing enzymes and act in concert to produce various steroids. In the central fasciculata zone (where the bulk of cortisol is produced) and elsewhere, steroidogenesis is greatly stimulated by exposure to $\mathrm{ACTH}$, which acts primarily by elevating cAMP and protein kinase A (PKA) activity. In 1971, Garren et al. (3) proposed that the effects of ACTH and cAMP are mediated by one or more labile proteins that turn over with a half-time of about 3 minutes. In early spectroscopic studies of P450scc in isolated mitochondria (4), it became clear that cholesterol binding by this cytochrome is enhanced by ACTH treatment. Because this change parallels an increase in a kinetically defined pool of rapidly metabolized cholesterol, these data indicated that the key action of ACTH is to make cholesterol available to the cytochrome (4). Further findings, indicating that P450scc is active prior to hormone treatment and that ACTH stimulates steroidogenesis only when the mitochondria are physically intact, were consistent with this idea and suggested that delivery of cholesterol across the mitochondrial membranes was limited and could be increased by ACTH treatment.

Later work (5) tied these threads together by showing that the labile protein factor predicted by Garren et al. (3) is required for cholesterol transfer from the outer mitochondrial membrane (OMM) to the inner mitochondrial membrane (IMM), where P450scc resides. Inhibition of protein synthesis in the presence of 
ACTH does not affect delivery of cholesterol to mitochondria but results in accumulation of cholesterol in OMM and a corresponding loss of IMM cholesterol. Cholesterol-P450scc complexes then cease to form, and pregnenolone synthesis is suppressed. The cytochrome itself is not affected by this treatment, since even mild mitochondrial disruption, which relieves the barrier to intermembrane transfer of cholesterol, restores steroidogenesis. Cholesterol transport between the mitochondrial membranes thus became recognized as a limiting, hormonally regulated process (4). This view received further support from work with relatively soluble hydroxycholesterol analogs, which can be metabolized to steroids without any activation process. Because such compounds are not constrained by membrane barriers, their turnover to pregnenolone is intrinsically rapid and cannot be further enhanced by ACTH.

These findings suggested that intermembrane transfer of cholesterol, the key control point for steroid biosynthesis, requires new synthesis of one or more proteins that become phosphorylated in response to cAMP. In 1983 and in subsequent papers, Orme-Johnson and colleagues identified a set of rapidly degraded 30-kDa mitochondrial phosphoproteins, collectively identified as phosphorylated p30 StAR (pp30) (6). These proteins are derived from a $\mathrm{p} 37$ precursor, which is phosphorylated in response to cAMP and is processed in the mitochondria to the pp30 form. Like the putative labile factor required for steroidogenesis, pp30 disappears rapidly after addition of the translational inhibitor cycloheximide (CHX). To explain the very rapid loss of activity in the presence of CHX, Orme-Johnson and colleagues proposed that the p37 protein is only effectively phosphorylated during translation, prior to entry into the inner mitochondria where pp30 is then formed (6). This model predicted that any loss of pp30 through intramitochondrial dephosphorylation could only be restored through a new cycle of synthesis. Later work does not support a role for dephosphorylation of pp30 in the rapid inactivation of cholesterol metabolism when protein synthesis is blocked. Nevertheless, other aspects of p37's itinerary and fate appear to match Orme-Johnson and colleagues' early predictions quite closely.

The StAR gene, cloned in 1994 by Clark et al. (7), encodes the $\mathrm{p} 37$ precursor protein identified by OrmeJohnson and colleagues. The properties of this unusual protein account for many of the anomalous characteristics of mitochondrial cholesterol metabolism. As predicted, StAR increases cholesterol metabolism and carries an $\mathrm{N}$-terminal mitochondrial targeting sequence. StAR is readily converted to a form that lacks the $68 \mathrm{~N}$-terminal amino acids and that corresponds to the previously reported $\mathrm{p} 30$ protein ACTH via cAMP and PKA generates $\mathrm{Pp} 30$. Consistent with earlier biochemical work that had shown pp30 to be regulated by calcium (8) as well as cAMP, StAR also contains consensus sequences for calmodulin-dependent kinases and PKA. As discussed below, the subsequent discovery that human CAH (a disease in which glucocorticoid production is greatly diminished) results from loss-of-function mutations in the StAR gene (9) established the essential role of this protein for normal adrenal steroidogenesis. This pathology is paralleled in StAR-null mice (10).

\section{Pre- and post-StAR events are hormonally regulated}

Cholesterol metabolism in normal adrenal cortex cells is acutely regulated by ACTH stimulation, rising appreciably within 3 minutes of treatment and peaking within 10-15 minutes (3). Defects in either PKA or G protein coupling, as seen in mutant mouse adrenal cell sublines, block this response by preventing cAMP formation (11). Other signaling pathways playing key roles in adrenal responses to ACTH include lipoxygenase activation (12) and, at least in adrenal fasciculata cells, stimulation mediated by receptors for IGF1, retinoids, and thyroid hormone; several cytokines, conversely, can suppress production of steroid hormones by these cells (13). For the most part, I will focus here on the mechanisms of acute adrenal fasciculata responses to cAMP and its analogs, which are generally shared with testicular and ovarian cells. It is interesting to note, however, that StAR regulation in another adrenal steroidogenic cell type, the glomerulosa cell, responds via alternative pathways involving $\mathrm{Ca}^{2+}$ stimulation (8).

While the unique CHX-sensitivity of adrenal cholesterol metabolism has drawn attention to mitochondrial transfer processes, the ACTH regulation of adrenal cells is equally dependent on the ability of cAMP to stimulate the rapid flow of cholesterol between the plasma membrane, cytoplasmic pools, and mitochondria. Typically, extramitochondrial transfer is limiting, thus preventing a build-up of OMM cholesterol (14), but such a build-up can occur when mitochondrial transfer is selectively compromised, for example following CHX treatment.

In rodent adrenal cells, the stimulation of cholesterol transfer to inner mitochondrial P450scc peaks within 10 minutes of ACTH treatment, prior to a substantial rise in StAR mRNA and protein (15). Thus, StAR rapidly ceases to be rate-limiting for steroidogenesis in these cells. On the other hand, in cultured human adrenal cells, as in testis MA10 cells, this increase in cholesterol metabolism is much slower $(16,17)$ and for several hours parallels StAR synthesis. However, even in these cells, StAR levels can continue to rise after a peak cholesterol metabolism is attained, indicating that some other component must become rate limiting.

Despite the high expression of P450scc in the adrenal cortex mitochondria, the availability of this enzyme can become limiting for steroid formation after strong ACTH stimulation (4). Expression of P450scc is itself regulated by ACTH and CAMP, and this response probably contributes more than has been recognized to the activation of cholesterol metabolism following exposure to hormone. Because the adrenal IMM lipid environment is rapidly altered by ACTH, exposure to this hormone can greatly alter cytochrome interactions with cholesterol (17), independent of any change in IMM cholesterol levels. 
The earlier assumption that intrinsic P450scc activity is unaffected by ACTH rested largely on the high, hormone-insensitive turnover of hydroxycholesterol. This finding now requires some reconsideration in light of evidence that P450scc interactions with cholesterol, but not with hydroxycholesterols, are strongly affected by the lipid environment of the IMM. Cholesterol interacts strongly with the fatty acid chains of many phospholipids and is thereby constrained from interacting with P450scc. Cholesterol binding to $\mathrm{P} 450 \mathrm{scc}$ in lipid vesicles is greatly potentiated when the local membrane is rendered more fluid by the addition of FFAs (18). Cardiolipin, which enhances the activesite binding of cholesterol to cytochrome P450scc, likewise promotes steroid formation (19). Hydroxycholesterols bind P450scc approximately ten times more strongly than does cholesterol, and they are not affected by the presence of cardiolipin. Hence, the absence of a hormonal effect on hydroxycholesterol metabolism may still obscure a real effect of ACTH on the relatively weak binding of cholesterol to the cytochrome.

Intramitochondrial movement of cytochrome P450scc to incoming cholesterol may also contribute to steroid production. Unlike other microsomal cytochromes, P450scc lacks an N-terminal transmembrane sequence and readily dissociates from the membrane (20). This enzyme is free to exchange between membranes, and it does so more rapidly than cholesterol, as judged by in vitro experiments using phospholipid vesicles (18). As Parker, Chung, Orly, and colleagues recently found, adrenal mitochondria of cells from mice deficient in P450scc fail to form the typical highly vesicular structure characteristic of the active adrenal cortex (21), suggesting a unique and important role for P450scc in mitochondrial membrane dynamics. Vesiculation of the IMM follows ACTH stimulation of hormonally suppressed adrenal glands and takes about 12 hours, consistent with the time for appearance of P450scc (22).

Cholesterol metabolism by $\mathrm{P} 450 \mathrm{scc}$ is sensitive not only to the lipid environment within the mitochondria, but also to the steady supply of oxygen (the co-substrate of the side chain cleavage reaction) and lipoprotein cholesterol (23), both of which reach the adrenal gland through the bloodstream. Blood flow, in turn, depends on ACTH, which stimulates endothelin production (24). Because of the rapidity of cholesterol turnover by P450scc, the pools of available and cytochrome-associated cholesterol in adrenal mitochondria are usually low (25), although it appears to build up when blood flow to the gland is restricted. Acute stimulation of blood flow by ACTH may therefore contribute to cortisol synthesis by enhancing extracellular access of both cholesterol and oxygen to P450scc.

In addition, among its many effects on cholesterol metabolism in the adrenal gland, cAMP elevates the expression of the LDL receptor (26) and SR-BI (27), promotes cholesterol delivery to the mitochondria from the plasma membrane (28) and possibly from the late endosomes (29), and stimulates cholesterol esterase activity while inhibiting acyl-CoA:cholesterol acyltransferase
(ACAT) (30). This combination of events favors the release of cholesterol from stored CEs and its net accumulation in mitochondria $(3,31)$. The late endosomes have been implicated as critical to delivery from LDL as evidenced by the inhibitory effect of the steroidamine U18666A. NPC-1 and the StAR-related protein MLN64 are likely mediators of this step. The various routes by which endogenous and exogenous cholesterol can reach the mitochondrion are shown in Figure 1.

\section{StAR can function at the OMM}

The site at which StAR acts to facilitate cholesterol metabolism by P450scc has been a matter of considerable debate and confusion. The 68-amino acid N-terminal sequence of $\mathrm{p} 37 \mathrm{StAR}$ directs the protein into the inner mitochondria, where specialized proteases generate the p30 form of the protein. Hormonal stimulation results in PKA-dependent phosphorylation of $\mathrm{p} 37$, generating pp30 StAR $(6,7,32-33)$, which accumulates within the mitochondrial matrix. Controversy has arisen because there is clear evidence from well-defined model systems that p37 StAR, interacting exclusively with the OMM, can mediate cholesterol transfer to IMM P450scc (34-36). Paradoxically, there is equally clear evidence that inner mitochondrial proteolysis in functioning adrenal cells is essential to cholesterol fluxes (33) and that high cholesterol transfer rates can be sustained after complete processing of pp37 to pp30. These seemingly incompatible findings probably reflect crucial differences between the experimental models, specifically the action of components limited to adrenal cells that permit pp30 to function within the IMM. In addition, it may be that different mechanisms of cholesterol transport predominate in different cell types.

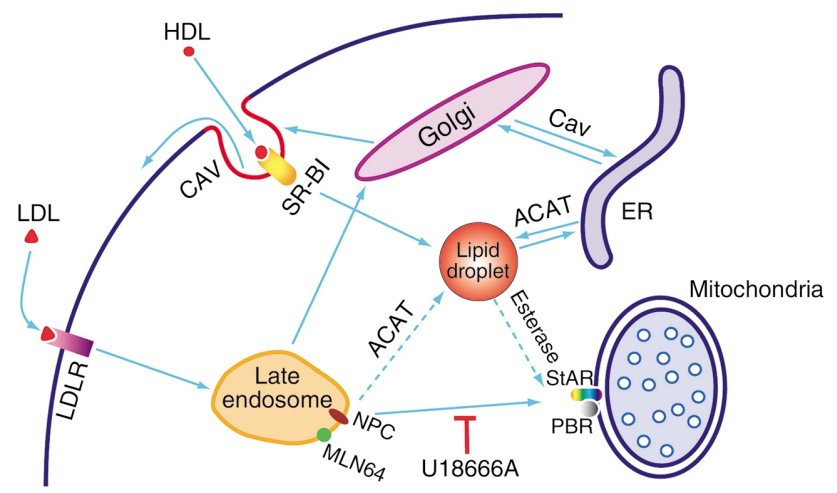

Figure 1

Mechanisms of cholesterol transfer to adrenal mitochondria. Steroidogenic cholesterol in adrenal cells transfers to PBR and then StAR in the mitochondria from the plasma membrane. Cholesterol is taken up from both $\mathrm{LDL}$ receptors and apoA/HDL receptors (SR-BI) in caveolin-rich (Cav-rich) domains. Late endosomes mediate this transfer to the mitochondria via the activities of NPC-1 (which is inhibited by U18666A) and possibly the StAR-like protein MLN64. Acyl-CoA:cholesterol acyltransferase (ACAT) converts free cholesterol derived from organelles (e.g., endosomes and endoplasmic reticulum (ER)) to the CEs that represent the predominant components of lipid droplets. Blue arrows indicate the routing of cholesterol into and through the cell. StAR is represented at the OMM and IMM, respectively, in its p37 and p30 forms. 


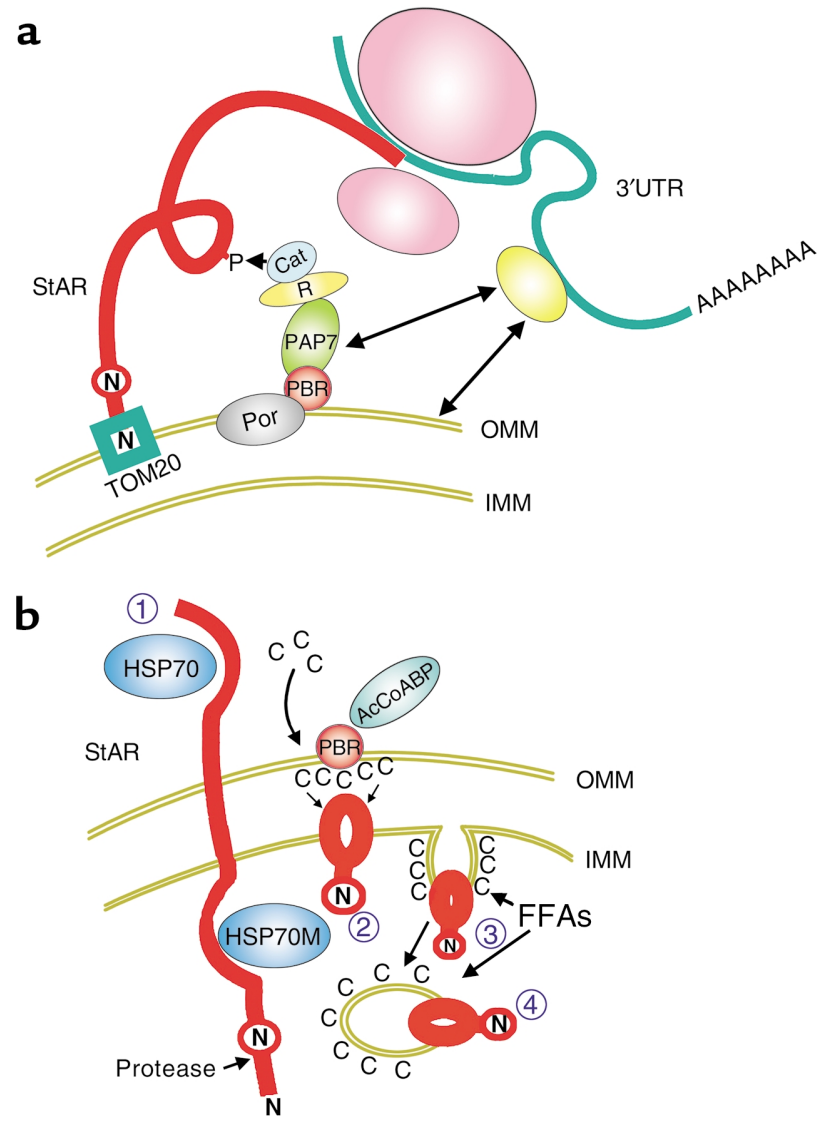

The model studies have relied on two types of experiments, which differ in important aspects with respect to regulation of cholesterol transport. First, recombinant StAR has been transfected into COS-1 cells that have been made functional for steroidogenesis through transfection of a chimeric P450scc/reductase molecule. COS- 1 cells are not normally steroidogenic and presumably lack many of the specialized components of the adrenal cholesterol processing system. Indeed, the transfected COS-1 mitochondria appear completely different from adrenal mitochondria and sustain only low rates of cholesterol metabolism in spite of high StAR expression (34). The second model system involves addition of extracts containing recombinant StAR to steroidogenic mitochondria. Here, the mitochondria usually come from MA10 cells that have not been stimulated by cAMP. These mitochondria have low basal activity for cholesterol metabolism and, again, probably lack key components, some of which will be discussed below.

In COS-1 cells, StAR can enhance cholesterol metabolism independent of the $\mathrm{N}$-terminal targeting sequence (34) that directs it to the OMM receptor TOM20 $(36,37)$ and then through the IMM, which initiates protein import into this organelle. Indeed, a COS-1 cell extract containing p30, which lacks this targeting sequence, is almost as effective as extracts expressing P37 in mediating cholesterol transfer into isolated MA10 mitochondria (37). These findings suggest that StAR can act on the OMM (34-36).

\section{Figure 2}

Models for StAR localization and cholesterol transfer into the mitochondrion. " $N$ " indicates the amino-terminus of the cytoplasmic p37 form of StAR; the circled " $N$ " indicates the amino-terminus of the processed mitochondrial form of StAR, Pp30. (a) StAR localization. Newly synthesized p37, shown here being translated by a cytoplasmic ribosome, becomes associated through its $\mathrm{N}$-terminal sequence with the OMM surface protein TOM20. Effective phosphorylation of $\mathrm{p} 37$ by the catalytic unit of PKA (Cat) is facilitated by interaction of the regulatory subunit $(R)$ with the adapter protein PAP7. This protein, in turn, binds to the PBR in the OMM, a partner with porin (Por). Interactions between the StAR mRNA $3^{\prime}$-untranslated region (3'UTR) and OMM proteins may also help target nascent StAR to the mitochondrion. (b) Transfer of StAR to the inner mitochondrion and delivery of OMM cholesterol to cytochrome P450scc. In step 1, following interaction with TOM20, pp37is denatured with assistance from cytosolic HSP70 complex and then extruded across the OMM and IMM. This process requires the proton gradient across the IMM and the action of mitochondrial matrix HSP70 (HSP70M). A protease cleaves pp37 to pp30, which is then integrated into the IMM. In step 2, cholesterol (C) transfers to pp30 from outer membrane domains formed by PBR, which is activated by acylCoA-binding protein (AcCoABP). In step 3, activated membrane regions, which contain high levels of FFAs, accept the transfer of cholesterol from the OMM cholesterol-rich domains generated around PBR. AcCoABP may also participate in the transfer of acyl-CoA to the IMM, where mitochondrial thioesterase (MTE) hydrolyzes this compound to FFA. In step 4, pp30 facilitates the relocation of cholesterol to p450ssc, which is distributed throughout the highly extended IMM, and possibly separate matrix vesicles.

Indeed, experiments with synthetic phospholipid membranes confirm that $\mathrm{p} 30$ and $\mathrm{p} 37$ are each capable of mediating transfer of cholesterol between mitochondrial membranes (38).

The effectiveness of outer membrane activity by StAR has been recently substantiated by an elegant series of experiments in which StAR was linked to components of the mitochondrial protein import system (36). These experiments showed that chimeric StAR, linked to the outer membrane TOM20 but not to intermembrane or inner mitochondrial channel (TIM9 or TIM18) proteins, effectively activates cholesterol metabolism in isolated basal MA10 mitochondria. Thus, StAR clearly can mediate cholesterol transfer when linked to the outer membrane of MA10 mitochondria. It should be noted that these experiments do not exclude a role for in the IMM, since, for example, these isolated MA10 cell mitochondria might fail to support StAR activity at this site. As discussed below, the proper regulation of IMM FFAs may be one key factor missing in COS cells and isolated MA10 mitochondria.

\section{StAR processing is preferred for high cholesterol fluxes}

There are several reasons to question the relevance of the OMM as the major site of StAR action in adrenal cells and, perhaps, in certain other cell types. Some of these concerns center on the properties of COS-1 cells. Thus, unlike in specialized steroidogenic cell types, cholesterol turnover in COS- 1 cells is relatively 
insensitive to changes in the level of StAR expression (C. Jefcoate, unpublished data). This finding may explain why transfected p30, which is broadly distributed in the COS- 1 cells, can be as effective as mitochondrially localized p37 StAR. COS-1 cells also process p 37 to p 30 much more slowly than do adrenal cells, thus providing more opportunity for an OMM mechanism to operate (7).

In addition, several well-established features of adrenal StAR biosynthesis appear inconsistent with the extramitochondrial StAR hypothesis. First, p37 turns over so quickly that peak cholesterol transfer occurs at a point when $\mathrm{p} 37$ can scarcely be detected in steroidogenic cells, particularly MA10 cells (33). Second, intramitochondrial processing is essential to StAR activity. Thus, the mitochondrial proteinase inhibitor orthophenanthrolene and the protein uptake inhibitor CCCP each block pp30 formation and prevent cAMP activation of cholesterol metabolism. While it is difficult to exclude other effects of these inhibitors, it is striking that they have no effect on cholesterol metabolism after sufficient pp30 has been generated (33). These experiments also show that once even modest amounts of new pp30 are available in the IMM, activated cholesterol metabolism becomes completely independent not only of processing, but also of both cAMP and protein synthesis.

While p37 certainly has activity when retained at the OMM, pp30, which forms very rapidly in the IMM, may therefore be the major contributor to enhanced cholesterol uptake to P450scc. There is reason to believe that pp30 StAR, acting within the mitochondrion, is additionally needed to expedite cholesterol entry into the IMM, where multiple proteins involved in respiration and ATP efflux reside. In mitochondria of typical, nonsteroidogenic cells, cholesterol is largely excluded from the IMM, suggesting that this lipid can be detrimental to respiratory functions (39). For instance, incubation of hepatocyte mitochondria with albumin-bound cholesterol can cause a tenfold increases in the OMM cholesterol and even induce the deposition of intermembrane cholesterol droplets while failing to increase IMM cholesterol. In adrenal cells, conversely, cholesterol can equilibrate between the $\mathrm{OMM}$ and IMM following ACTH treatment in the presence of a P450scc inhibitor $(5,14)$. ACTH-stimulated expansion of the IMM and mobilization of P450scc may represent specialized responses of these mitochondria, allowing cholesterol pools to be physically segregated from the electron transport chain and other normal IMM proteins.

The unusually extensive and vesiculated IMM in adrenal cells, much of which does not have direct access to the OMM, poses a particular problem for efficient cholesterol delivery to P450scc. Primary intermembrane cholesterol transfer may only occur to regions of the IMM that juxtapose the OMM. As argued below, pp30 StAR may be key to a secondary step in which substrate cholesterol is delivered to P450scc at locations in the inner mitochondrion that are well removed from these peripheral IMM sites.

\section{Intramitochondrial activation, deactivation, and disposal of StAR}

While p37 StAR can clearly mediate cholesterol uptake into COS-1 cell mitochondria, its ability to do so in steroidogenic cells in vivo is likely constrained by the small amount of p37 associated with the OMM at steady state and by the distinctive biochemical and morphological features of mitochondria in these specialized cells. An alternative hypothesis (presented in Figure 2) holds that StAR functions as pp30 via interaction with the inner surface of the IMM. This mechanism is consistent with well-established aspects of StAR activation and disposal in active adrenal cells.

Newly synthesized StAR is essential for cholesterol transfer even when large amounts of multiple forms of variously modified StAR are present in the mitochondria prior to stimulation of the adrenal cells (33). The conversion of newly formed p37 to the p30 form is highly efficient and reaches a steady state between formation and processing within 5 minutes, consistent with very rapid turnover of p37 ( $t_{1 / 2}$ approximately $1-2$ minutes). cAMP has no measurable effect on these rates, but maximum stimulation ensures near-complete conversion, presumably by promoting the extramitochondrial phosphorylation of p37. The inhibition of ACTH-stimulated cholesterol metabolism by protein synthesis inhibitors, even when there is abundant IMM pp30, is attributed to the rapid loss of activity of this protein following synthesis. Each molecule of newly synthesized pp30 StAR is estimated to mediate the transfer of 400 cholesterol molecules per minute (33). However, most of the pp30 that persists in the mitochondrion, including the bulk of the older molecules, may not be properly localized to contribute to intermembrane cholesterol transfer. Based on immunogold labeling experiments, StAR is found predominantly around the matrix vesicles in normal adrenal glands $(40,41)$. At this site, StAR seems unlikely to affect this process. Conversely, newly synthesized protein may be present preferentially in IMM segments that are proximal to the OMM.

Orly has recently provided evidence that inner mitochondrial p30 turns over slowly via a two-step process (42). Pulse-labeling experiments show that p30 is blocked within 2 hours after transfer to the IMM by selective protease inhibitors that subsequently become ineffective. Orly suggests that the inhibitor-sensitive StAR pool and the protease are located close to the site where p30 first enters the IMM. StAR at this site likely represents the IMM pool that is active in facilitating uptake from the OMM.

\section{Deficiency of StAR function in relation to StAR structure}

The mutations in StAR that lead to human CAH are remarkably informative about StAR function (9), since the disease alleles lead to C-terminal truncation or affect mutation at amino acids known to contribute to cholesterol transfer. A mutant carrying a nonsense mutation 28 amino acids from the normal C-terminus is seen in 80 percent of Japanese cases, and other mis- 
sense and short deletion alleles affecting the C-terminal region are expressed but inactive in stimulating cholesterol metabolism. The positions of these mutations fit well with key structural sites predicted by the recent crystal structure of the related MLN64 (43), which shares with StAR a conserved structure termed a StAR-related lipid transfer (START) domain. The key feature of the structure, which presumably is shared by StAR, is a central channel, open at both ends, that can fit a cholesterol molecule. This presumptive cholesterol channel has positive and negatively charged amino acids at one end, including D169, which is mutated in some CAH patients.

Recent work using fluorescence transfer shows that the cholesterol derivative NBD-cholesterol binds with high affinity $\left(K_{\mathrm{d}}=32 \mathrm{nM}\right)(44)$ and that StAR activity alters the lipid mobility of the surrounding membrane, as detected with fluorescent probes. START domains are also found in phosphatidylcholine transfer protein and diverse other proteins that bind phospholipids (see Maxfield, this Perspective series, ref. 45). However, two key residues that are shared with MLN64 (Asp144 and Asn148) are absent in other family members. The relatively polar StAR protein associates very strongly with mitochondrial membranes, suggesting possible phospholipid interactions.

Disruption of the StAR gene in mice produces a similar phenotype to $\mathrm{CAH}$ (10). ACTH-stimulated glucocorticoid output is lowered about 20 -fold. Loss of feedback inhibition by glucocorticoids in the pituitary causes $\mathrm{ACTH}$ release to rise with a consequent stimulation of lipoprotein receptor-mediated uptake of CEs and hyperplasia. The enormous lipid accumulation is ultimately toxic to the gland. Fortunately, the corresponding clinical effects can be reversed by early treatment of children with glucocorticoids, and this treatment is also effective in StAR-deficient mice. In males of this strain, testosterone production is low and large amounts of lipid accumulate in the testis, where hyperplasia also ensues due to the elevated pituitary response. The ovaries are normal at birth but also exhibit interstitial lipid accumulation and slow maturation. Residual, StAR-independent cholesterol metabolism in the adrenal glands is clearly measurable and seems to be sufficient for the slower metabolism to estradiol in the ovaries.

\section{The peripheral benzodiazepine receptor is essential for StAR activity}

Like StAR, the peripheral benzodiazepine receptor (PBR) is strongly implicated in mitochondrial cholesterol uptake. PBR is located in the outer membrane of most mitochondria and is elevated in steroidogenic cells. Developing a model for steroidogenesis that integrates the information about both of these proteins has proved a significant challenge.

PBR associates with a member of the porin family of outer membrane channel proteins. When PBR is activated by drug agonists or by the adrenal protein acylCoA-binding protein (also called DB1 and endozepine), it alters the porin's permeability and thereby affects a variety of mitochondrial functions. PBR agonists also stimulate cholesterol metabolism in adrenal and testis cells, while antagonists prevent the induction of mitochondrial cholesterol transport and inhibit cAMP-stimulated steroidogenesis (46). Deletion of PBR severely compromises cholesterol metabolism, which can be restored by transfection of cells with PBR cDNAs. Agonist binding causes relocation of the outer membrane cholesterol to PBR, which binds the lipid via a defined sequence distinct from a START domain. The key role for PBR in cholesterol metabolism in adrenal cortex cells is substantiated by the observation that ginkgolide treatment suppresses both cholesterol metabolism and PBR expression without affecting StAR expression (47). Also suggestive is the finding that adrenal cholesterol metabolism in the neonatal rat almost disappears between days 5 and 15 in parallel with a loss of PBR (48); StAR shows only modest changes in expression over this developmental period.

Recombinant StAR and PBR can be cross-linked in COS-1 cells (49), where StAR slowly accumulates in the p37 form (7). This physical interaction has been suggested to be important for p37 entry into the mitochondria and ultimately for cholesterol import (50). While neither PBR nor the upstream regulator, DBI, is substantially elevated by ACTH treatment, the recent identification of a protein, termed PAP7, that binds both PBR and PKA in steroidogenic cells suggests a possible pathway leading to phosphorylation of p37 StAR (Figure 2a; see also ref. 51). A multiprotein complex centered on PAP7 may also mediate the interaction between StAR and PBR. Indeed, suppression of PAP7 expression using an antisense approach prevents CAMP stimulation of cholesterol metabolism in MA10 cells.

Much has been made of the difficulty in transferring cholesterol across an aqueous intermembrane space, but, as discussed above, it may be more critical to overcome the resistance of the inner membrane to take up cholesterol. In the model shown in Figure 2a, activated pp37 moves through the membranes in a denatured form and then refolds after cleavage in the matrix to generate $\mathrm{pp} 30$. This process is assisted by HSP70, which acts continuously as pp30 emerges through the IMM. StAR is believed to readily adopt a molten globule structure (52), which may be stabilized by HSP70, much as HSP interactions with steroid receptors facilitate steroid binding (53) (Figure 2b, step 2). This StAR conformation may be necessary to provide a strong interaction with the IMM. Activated membrane regions may accept the transfer of cholesterol from the OMM cholesterol-rich domains generated around PBR (step 3). (The role of FFAs in the activation of the IMM is discussed below.) Finally, pp30 facilitates the relocation of cholesterol from the IMM sites proximal to the OMM to the matrix vesicle sites (step 4). The translocation of StAR to the mitochondrial matrix (step 4) could be an essential part of the internalization of mitochondrial cholesterol, particularly in the more active adrenal cortex cells, where the bulk of P450scc is in these matrix vesicles. It is this final relocation step in the pathway that may cause the sensitivity to protein synthesis inhibitors. The inabili- 
ty of the mitochondrion to replenish StAR as it is lost from proximal IMM sites could account for the blockade of ACTH-stimulated cholesterol transfer seen within minutes following $\mathrm{CHX}$ treatment.

\section{Stimulation of StAR activity by mitochondrial FFAs}

Recent work indicates that StAR is critically sensitive to the presence of FFAs. A novel mechanism has come to light whereby long-chain fatty acids released from CE hydrolysis can accumulate within the mitochondria of steroidogenic cells and stimulate cholesterol metabolism. This pathway, which is probably not found in other cell types, could help explain the inconsistent functional data that have plagued this field.

The Podesta laboratory has recently established that transfer of fatty acyl-CoA to the inner mitochondria is essential for optimal StAR activity. The mitochondrial thioesterase (MTE; also called ARTISt), which hydrolyzes acyl-CoA to release FFAs, carries a mitochondrial targeting sequence and is found in vivo in the inner mitochondrion (54). This enzyme is presumed to release arachidonic acid (AA) and other FFAs inside this organelle. Because linoleic and oleic acids are just as effective as AA at overcoming a block in steroid formation imposed by MTE inhibition (C. Jefcoate and E.J. Podesta, unpublished data), it appears that StAR activation requires fatty acids per se, rather than prostaglandins, leukotrienes, or other AA oxidation products.

Fatty acyl-CoAs derived from the hydrolysis of cytoplasmic CEs bind to the acyl-CoA-binding protein, which can bind and may thereby activate PBR (46). This interaction favors the accumulation of fatty acids near StAR, perhaps by promoting fatty acyl CoA transfer into the inner mitochondria. While the mitochondrial fate of these fatty acids is clearly critical to the operation of the cholesterol uptake pathway, the mechanism by which fatty acids promote steroidogenesis has not been established. Possibly, the increase in membrane fluidity in the presence of these fatty acids favors the interaction of cholesterol with StAR or P450scc $(18,44)$. Podesta has pointed out that involvement of fatty acyl CoA and MTE would be compatible with the central role of PBR in cholesterol transport to the IMM (54).

\section{Shaping up for StAR}

An often overlooked component of the acute adrenal response is the extraordinarily fast cell contraction that is essential for the hormonal activation of cholesterol metabolism. Adrenal cells and testis in culture completely round up as a result of cytoskeletal structural changes within a few minutes following ACTH stimulation (55). This change in cell shape and the attendant changes in cytoskeletal organization play an important role in the acute stimulation of cholesterol metabolism and the induction of StAR activity by ACTH and cAMP. Cytoskeletal disruption, which prevents this response, blocks cholesterol transfer to mitochondria (56).

Notably, the cytoskeleton-associated protein paxillin, a component of the focal adhesion complex, undergoes cAMP-dependent dephosphorylation with a half-time of about 5 minutes in parallel with the cell rounding in culture (55). PTPase inhibitors that block paxillin dephosphorylation (54) also substantially inhibit the acute stimulation of cholesterol metabolism. The same effects of ACTH on paxillin dephosphorylation are seen in adrenal glands in vivo, although the relationship to steroidogenesis in the tissue remains to be determined. This change is generated via cAMP/PKA activation of a phosphotyrosine phosphatase, SHP2 $(55,64)$, whose time course is similar to that of paxillin dephosphorylation.

\section{Regulation of StAR expression}

Two species of StAR mRNA (1.6 and $3.5 \mathrm{~kb}$ ) are stimulated by cAMP in rodent steroidogenic cells (58). In human but not in rodent cells, the short form seems to predominate both before and after stimulation (57). The StAR gene has seven exons $(58,59)$, with exon 7 containing four sites for polyadenylation; these sites give rise to alternative mRNA forms. These predominant mRNA species differ only in their site of polyadenylation, but they can be differentially regulated, since the longer $3^{\prime}$-untranslated region contains AU-rich elements that are recognized specifically by cytoplasmic proteins (59). Stabilization of labile mRNA by such factors provides a much faster response than is possible by transcriptional induction, a strategy that is used by early response genes and cytokines to upregulate gene expression within 15 minutes $(60,61)$.

StAR expression is also regulated by the transcription factor SF-1, for which several promoter elements have been identified (13). Expression of StAR and other steroidogenic factors is lost in SF-1-deficient mice. The nuclear regulatory protein DAX1 inhibits StAR promoter activity by binding in the region of the proximal SF-1 site (62). SF-1 is not directly activated by PKA phosphorylation but can be activated by mitogen-activated protein kinase (MAPK) (63). CEBP $\beta$ also plays an important role in cAMP regulation of $\operatorname{StAR}(13,64)$.

StAR transcription is stimulated not only through ACTH and cAMP, but also in response to AA and cholesterol levels (62), and it is clearly integrated in this way to other components of the cholesterol processing machinery. Hormonal stimulation of StAR causes a rapid release of AA from phospholipids, which is apparently critical for the activation of StAR transcription by cAMP (65). This release of AA is mediated by phospholipase $A$ and leads to formation of a lipoxygenase product. AA hydroperoxides stimulate StAR expression directly, while lipoxygenase inhibitors prevent enhanced transcription. This process is clearly distinct from the acute requirement for FFAs in the mitochondria, which involves a broader range of fatty acids. The mechanism whereby AA hydroperoxides cooperate with cAMP remains to be elucidated. Cholesterol depletion stimulates StAR transcription through participation of SREBP, which is elevated in the nucleus through a cholesterol-sensitive microsomal cleavage to a 68 -kDa nuclear regulatory factor (66). Various mechanisms of posttranscriptional and transcriptional StAR regulation are summarized in Figure 3. 


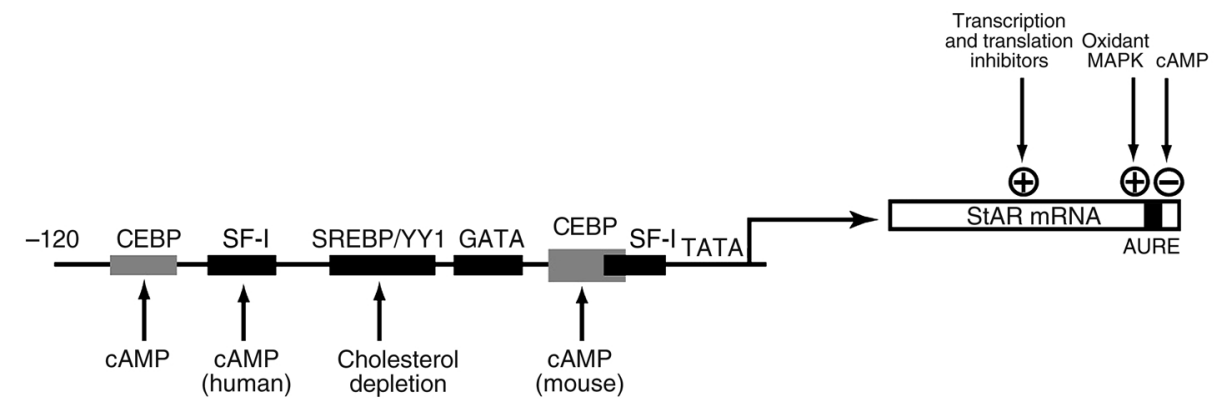

\section{Figure 3}

Regulation of StAR mRNA expression. StAR mRNA expression is determined by the balance between transcription and mRNA turnover, each of which is regulated by multiple factors. Promoter elements and mRNA sequence elements are shown that are subject to regulation by physiological changes, such as hormonal stimulation (which increases CAMP levels) and cholesterol depletion (which activates SREBP). cAMP and oxidant-activated MAP kinases exert negative and positive effects respectively on mRNA stability, possibly via the StAR AU-rich element (AURE). StAR mRNA stability is also regulated by suppression of transcription or translation. Stabilization of an otherwise rapidly degraded mRNA is a regulatory mechanism that allows for extremely rapid and sensitive control of gene expression.

\section{StAR: not the last word}

While we now have an excellent understanding of $\mathrm{CAH}$, relatively little is known about the role of StAR during the normal acute regulation in human adrenal cells. Available human cell models respond very slowly to cAMP (16) relative to the adrenal gland in vivo, and even in rodent models, most studies have been carried out under maximum stimulatory conditions. More information is needed on adrenal cell responses to low levels of ACTH, as well as to various cytokines and thyroid and retinoid hormones (67-69). Much also needs to be determined about the signaling that links these activators and suppressors to the key regulators, particularly components of the MAPK pathways (70). Many of these effects are highly tissue- and species-selective and may be sensitive to diet and circadian control.

High cholesterol fluxes through adrenal cortex cells are subject to regulation at multiple points and by diverse signaling processes affecting cytoplasmic transfer and transfer into the mitochondria. These processes need to be balanced in order to maintain mitochondrial access to cholesterol while at the same time avoiding an adverse accumulation of cholesterol in the mitochondria. Cholesterol uptake and transfer to the mitochondria are hormonally regulated at multiple levels, including lipoprotein receptors, CE hydrolysis, and endosome transport. Mitochondrial transfer from outer to inner membrane, while requiring StAR as an essential component, also shows a diversity of regulators and mechanisms.

This diversity of regulation is not surprising in view of the central role of glucocorticoid synthesis in integrating acute metabolic and immune responses and the adrenal gland's key function in organizing responses to rapid changes in environment. While I have focused above on the novel mitochondrial processes involving StAR, it is critical to recognize that these probably only become rate-limiting under unusual circumstances. These conditions have been generated by specific manipulations, including the genetic depletion of StAR and the complete inhibition of protein synthesis. MTE and PBR $(46,54)$ appear to be just as essen- tial as StAR for steroidogenesis, but the consequences of aberrant adrenal expression or pharmacological manipulation of these proteins are not well understood. Nevertheless, it seems clear that the mitochondrial process is both integrated and flexible, since it can respond to changes in fatty acid metabolism through MTE, to altered mitochondrial energy fluxes through $\mathrm{PBR}$, and to interruptions in protein synthesis through the rapid functional turnover of StAR.

The question of whether StAR functions as p37 in the OMM or as Pp30 in the IMM is not fully resolved, but it should be noted that these are not exclusive mechanisms and may either work together or be selectively preferred according to the circumstances. The OMM mechanism has been rigorously established, while the evidence for the IMM mechanism is dependent on strong circumstantial data. The mechanism that predominates in a given cell likely depends on biochemical context. OMM transfer could be favored when processing is slow, when the IMM is not unusually extended, or when MTE is absent. One or more of these conditions seem to apply in COS- 1 cells and in isolated mitochondria from unstimulated steroidogenic cells; as argued above, they probably do not apply in highly active adrenal or even testis cells. Which of these mechanisms act in the ovary and the brain (tissues with lower demand for steroid synthesis) is not yet clear.

Essentially all mechanistic experiments to date have focused on comparisons of the fully activated cells, but much remains to be learned about how these various processes function under different stimulation conditions, which may alter the balance between the various components. There may also be tissue-specific advantages of changes in the favored mechanism. For example, cholesterol transfer driven by an extramitochondrial p37 StAR can be regulated from the cytosol more readily than that driven by the intramitochondrial pp30. The latter, on the other hand, may introduce a greater sensitivity to mitochondrial energetics and ion balance.

Several methods have been developed for dissecting these processes. In the future, it will be important to focus on more specific methods of dissection under 
physiological conditions; for example, antisense and RNA interference to selectively deplete the various components. Unfortunately, the adrenal cortex remains a very enigmatic tissue, in part because cultures do not maintain the full physiological function. Even adrenal primary cultures lose a very large amount of the cholesterol flux seen in adrenal glands. Still, adrenal cells continue to be an important system for studying cholesterol transfer. The integration and cell specificity of these processes remain major challenges for future research.

1. Pollack, S.E., et al. 1997. Localization of the steroidogenic acute regulatory protein in human tissue. J. Clin. Endocrinol. Metabol. 82:4243-4251.

2. Bauer, M.P., Bridgham, J.T., Langenau, D.M., Johnson, A.L., and Goetz, F.W. 2000. Conservation of steroidogenic acute regulatory (StAR) protein structure and expression in vertebrates. Mol. Cell. Endocrinol. 168:119-125.

3. Garren, L.D., Gill, G.N., Masui, H., and Walton, G.M. 1971. On the mechanism of action of ACTH. Recent Prog. Horm. Res. 27:433-478.

4. Jefcoate, C.R., et al. 1973. The detection of different states of the P-450 cytochromes in adrenal mitochondria: changes induced by ACTH. Ann. N.Y. Acad. Sci. 212:243-261.

5. Privalle, C.T., McNamara, B.C., Dhariwal, M.C., and Jefcoate, C.R. 1987. ACTH control of cholesterol side-chain cleavage at adrenal mitochondrial cytochrome P450scc. Regulation of intramitochondrial cholesterol transfer. Mol. Cell. Endocrinol. 53:87-101.

6. Epstein, L.F., and Orme-Johnson, N.R. 1991. Regulation of steroid hormone biosynthesis. Identification of precursors of a phosphoprotein targeted to the mitochondrion in stimulated rat adrenal cortex cells. J. Biol. Chem. 266:19739-19745.

7. Clark, B.J., Wells, J., King, S.R., and Stocco, D.M. 1994. The purification, cloning, and expression of a novel luteinizing hormone-induced mitochondrial protein in MA-10 mouse Leydig tumor cells. Characterization of the steroidogenic acute regulatory protein (StAR). J. Biol. Chem. 269:28314-28322.

8. Elliott, M.E., Goodfriend, T.L., and Jefcoate, C.R. 1993. Bovine adrenal glomerulosa and fasciculata cells exhibit 28.5-kilodalton proteins sensitive to angiotensin, other agonists, and atrial natriuretic peptide. Endocrinology. 133:1669-1677.

9. Bose, H.S., Sugawara, T., Strauss, J.F.R., and Miller, W.L. 1996. The pathophysiology and genetics of congenital lipoid adrenal hyperplasia. International Congenital Lipoid Adrenal Hyperplasia Consortium. N. Engl. J. Med. 335:141870-141878.

10. Hasegawa, T., et al. 2000. Developmental roles of the steroidogenic acute regulatory protein (StAR) as revealed by StAR knockout mice. Mol. Endocrinol. 14:1462-1471.

11. Olson, M.F., Krolczyk, A.J., Gorman, K.B., Steinberg, R.A., and Schimmer, B.P. 1993. Molecular basis for the $3^{\prime}, 5^{\prime}$-cyclic adenosine monophosphate resistance of kin mutant Y1 adrenocortical tumor cells. Mol. Endocrinol. 7:477-487.

12. Yamazaki, T., Higuchi, K., Kominami, S., and Takemori, S. 1996. 15Lipoxygenase metabolite(s) of arachidonic acid mediates adrenocorticotropin action in bovine adrenal steroidogenesis. Endocrinology. 137:2670-2675.

13. Christenson, L.K., and Strauss, J.F., III. 2000. Steroidogenic acute regulatory protein (StAR) and the intramitochondrial translocation of cholesterol. Biochem. Biophys. Acta. 1529:175-187.

14. DiBartolomeis, M.J., Williams, C., and Jefcoate, C.R. 1986. Inhibition of ACTH action on cultured bovine adrenal cortical cells by 2,3,7,8-tetrachlorodibenzo-p-dioxin through a redistribution of cholesterol. J. Biol. Chem. 261:4432-4437.

15. Jefcoate, C.R., Artemenko, I.P., and Zhao, D. 2000. Relationship of StAR expression to mitochondrial cholesterol transfer and metabolism. Endocr. Res. 26:663-680.

16. Liu, P.H., Kahri, A.I., and Voutilainen, R. 1996. Expression of the steroidogenic acute regulatory protein $\mathrm{mRNA}$ in adrenal tumors and cultured adrenal cells. J. Endocrinol. 150:43-50.

17. Igarashi, Y., and Kimura, T. 1986. Adrenic acid content in rat adrenal mitochondrial phosphatidylethanolamine and its relation to ACTHmediated stimulation of cholesterol side chain cleavage reaction. J. Biol. Chem. 261:14118-14124.

18. Dhariwal, M.S., and Jefcoate, C.R. 1989. Cholesterol metabolism by purified cytochrome P-450scc is highly stimulated by octyl glucoside and stearic acid exclusively in large unilamellar phospholipid vesicles. Biochemistry. 28:8397-8402.

19. Lambeth, J.D., Kitchen, S.E., Farooqui, A.A., Tuckey, R., and Kamin, H.
1982. Cytochrome P-450scc-substrate interactions. Studies of binding and catalytic activity using hydroxycholesterols. J. Biol. Chem. 257:1876-1884.

20. Hanukoglu, I., Spitsberg, V., Bumpus, J.A., Dus, K.M., and Jefcoate, C.R. 1981. Adrenal mitochondrial cytochrome P-450 scc. Cholesterol and adrenodoxin interactions at equilibrium and during turnover. J. Biol. Chem. 256:4321-4328.

21. Ishii, T., et al. 2002. The roles of circulating high-density lipoproteins and trophic hormones in the phenotype of knock-out mice lacking steroidogenic acute regulatory protein. Mol. Endocrinol. In press.

22. Bornstein, S.R., Ehrhart-Bornstein, M., Guse-Behling, H., and Scherbaum, W.A. 1992. Structure and dynamics of adrenal mitochondria following stimulation with cotricotropin releasing hormone. Anat. Rec. 234:255-262.

23. Stevens, V.L., Aw, T.Y., Jones, D.P., and Lambeth, J.D. 1984. Oxygen dependence of adrenal cortex cholesterol side chain cleavage. J. Biol. Chem. 259:1174-1179.

24. Gaillard, I., et al. 2000. ACTH-regulated expression of vascular endothelial growth factor in the adult bovine adrenal cortex: a possible role in the maintenance of the microvasculature. J. Cell. Physiol. 185:226-234.

25. Jefcoate, C.R., and Orme-Johnson, W.H. 1975. Cytochrome P-450 of adrenal mitochondria. In vitro and in vivo changes in spin states. J. Biol. Chem. 250:4671-4677.

26. Kovanen, P.T., Goldstein, J.L., Chappell, D.A., and Brown, M.S. 1980. Regulation of low density lipoprotein receptors by adrenocorticotropin in the adrenal gland of mice and rats in vivo. J. Biol. Chem. 255:5591-5598.

27. Temel, R.E., et al. 1997. Scavenger receptor class B, type I (SR-BI) is the major route for the delivery of high density lipoprotein cholesterol to the steroidogenic pathway in cultured mouse adrenocortical cells. Proc. Natl. Acad. Sci. USA. 94:13600-13605.

28. Gocze, P.M., and Freeman, D.A. 1992. A cholesterol ester hydrolase inhibitor blocks cholesterol translocation into the mitochondria of MA10 Leydig tumor cells. Endocrinology. 131:2972-2978.

29. Watari, H., et al. 2000. NPC-1 containing compartment of human granulosa cells: a role in intracellular trafficking of cholesterol supporting steroidogenesis. Exp. Cell Res. 255:56-66.

30. Buhman, K.F., Accad, M., and Farese, R.V., Jr. 2000. Mammalian acylCoA:cholesterol acyltransferases. Biochem. Biophys. Acta. 1529:142-154.

31. Jamal, Z., Suffolk, R.A., Boyd, G.S., and Suckling, K.E. 1985. Metabolism of cholesteryl ester in monolayers of bovine adrenal cortical cells. Effect of an inhibitor of acyl-CoA:cholesterol acyltransferase. Biochim. Biophys. Acta. 834:230-237.

32. Stocco, D.M., and Clark, B.J. 1996. Regulation of the acute production of steroids in steroidogenic cells. Endocrinol. Rev. 17:221-224.

33. Artemenko, I.P., Zhao, D., Hales, D.B., Hales, K.H., and Jefcoate, C.R. 2001. Mitochondrial processing of newly synthesized StAR, but not total StAR, mediates cholesterol transfer to cytochrome P450 side chain cleavage enzyme in adrenal cells. J. Biol. Chem. 276:46583-46596.

34. Arakane, F., et al. 1996. Steroidogenic acute regulatory protein (StAR) retains activity in the absence of its mitochondrial import sequence: implications for the mechanism of StAR action. Proc. Natl. Acad. Sci. USA. 93:13731-13736.

35. King, S.R., et al. 1995. Steroid production after in vitro transcription, translation, and mitochondrial processing of protein products of complementary deoxyribonucleic acid for steroidogenic acute regulatory protein. Endocrinology. 136:5165-5176.

36. Bose, H.S., Lingappa, V.R., and Miller, W.L. 2002. Rapid regulation of steroidogenesis by mitochondrial protein import. Nature. 417:87-91.

37. Koehler, C.M. 2000. Protein translocation pathways of the mitochondrion. FEBS Lett. 476:27-31.

38. Kallen, C.B., et al. 1998. Steroidogenic acute regulatory protein (StAR) is a sterol transfer protein. J. Biol. Chem. 273:26285-26288.

39. Echegoyen, S., et al. 1993. Cholesterol increase in mitochondria: its effect on inner-membrane functions, submitochondrial localization and ultrastructural morphology. Biochem. J. 289:703-708.

40. Lehoux, J.G., et al. 1999. The in vivo effects of ACTH and sodium on the formation of different species of StAR in rat adrenal. Endocrinology. 140:5154-5164.

41. Ronen-Fuhrmann, T., et al. 1998. Spatio-temporal expression patterns of steroidogenic acute regulatory protein (StAR) during follicular development in the rat ovary. Endocrinology. 139:303-315.

42. Orly, J. 2002. Birth and Death of StAR - Easy come, easy go. Endocr. Res. In press.

43. Tsujishita, Y., and Hurley, H. 2000. Structure and lipid transport mechanism of a StAR-related domain. Nat. Struct. Biol. 7:408-414.

44. Petrescu, A.D., Gallegos, A.M., Okamura, Y., Strauss, J.F., III, and Schroeder, F. 2001. Steroidogenic acute regulatory protein binds cholesterol and modulates mitochondrial membrane sterol domain dynamics. J. Biol. Chem. 276:36970-36982.

45. Maxfield, F.R., and Wüstner, D. 2002. Intracellular cholesterol transport. 
J. Clin. Invest. 110:891-898. doi:10.1172/JCI200216500.

46. Papadopoulos, V. 1998. Structure and function of the peripheral benzodiazepine receptor in steroidogenic cells. Proc. Soc. Exp. Biol. Med. 217:130-142.

47. Amri, H., Dreiu, K., and Papadopoulos, V. 1997. Ex vivo regulation of adrenal cortical cell steroid and protein synthesis, in response to adrenocorticotropic hormone stimulation, by the Ginkgo biloba extract Egb 761 and isolated ginkgolide B. Endocrinology. 138:5415-5426.

48. Zilz, A., Castello, R., Papadopoulos, V., and Widmaier, E.P. 1999. Developmental expression of the peripheral-type benzodiazepine receptor and the advent of steroidogenesis in rat adrenal glands. Endocrinology. 140:859-864

49. West, L.A., et al. 2001. Steroidogenic acute regulatory protein and peripheral-type benzodiazepine receptor associated at the mitochondrial membrane. Endocrinology. 142:502-505.

50. Hauet, T., Liu, J., Li, H., Papadopoulos, V. 2002 Partners in cholesterol transport. Endocr. Res. In press.

51. Li, H., et al. 2001. Identification, localization, and function of PAP7: a peripheral-type benzodiazepine receptor and PKA (RI[alpha])-associated protein. Mol. Endocrinol. 15:2211-2228.

52. Christensen, K., Bose, H.S., Harris, F.M., Miller, W.L., and Bell, J.D. 2001. Binding of StAR to synthetic membranes suggests an active molten globule. J. Biol. Chem. 276:17044-17051.

53. Murphy, P.J., Kanelakis, K.C., Galigniana, M.D., Morishima, Y., and Pratt, W.B. 2001. Stoichiometry, abundance, and functional significance of the hsp90/hsp70-based multiprotein chaperone machinery in reticulocyte lysate. J. Biol. Chem. 276:30092-30098.

54. Maloberti, P., et al. 2000. Regulation of arachidonic acid release in steroidogenesis: role of a new acyl-CoA thioesterase (ARTISt). Endocr. Res. 26:653-662.

55. Rocchi, S., Gaillard, I., Obberghen, E.V., Chambaz, E.M., and Vilgrain, I 2000. Adrenocorticotrophic hormone stimulates phosphotyrosine phosphatase SHP2 in bovine adrenocortical cells: phosphorylation and activation by camp-dependent protein kinase. Biochem. J. 352:483-490.

56. Hall, P.F. 1995. The roles of microfilaments and intermediate filaments in the regulation of steroid synthesis. J. Steroid Biochem. Mol. Biol. 55:601-605.

57. Paz, C., Cornejo Maciel, F., Poderoso, C., Gorostizaga, A., and Podesta, E.J. 2000. An ACTH-activated protein tyrosine phosphatase (PTP) is modulated by PKA-mediated phosphorylation. Endocr. Res. 26:609-614 58. Ariyoshi, N., Kim, Y.C., Artemenko, I., Bhattacharyya, K., and Jefcoate,
C.R. 1998. Characterization of rat StAR gene that encodes the predom inant 3.5-kilobase mRNA. J. Biol. Chem. 273:7610-7619.

59. Sugawara, T., Lin, D., and Holt, J.D. 1995. Structure of the human steroidogenic acute regulatory protein (StAR) gene: StAR stimulates mitochondrial cholesterol 27-hydroxylase activity. Biochemistry. 34:12506-12512.

60. Capowski, E.E., Esnault, S., Bhattacharya, S., and Malter, J.S. 2001. Y boxbinding factor promotes eosinophil survival by stabilizing granulocytemacrophage colony-stimulating factor mRNA. J. Immunol. 167:5970-5976.

61. Steitz, J.A., and Brennan, C.M. 2001. HuR and mRNA stability. Cell. Mol. Life Sci. 58:266-277.

62. Ito, M., Yu, R., and Jameson, J.L. 1997. DAX-1 inhibits SF-1-mediated transactivation via a carboxy-terminal domain that is deleted in adrenal hyoplasia congenita. Mol. Cell. Biol. 17:1476-1483.

63. Hammer, G.D., et al. 1999. Phosphorylation of the nuclear receptor SF-1 modulates cofactor recruitment: integration of hormone signaling in reproduction and stress. Mol. Cell. 3:521-526.

64. Christenson, L.K. Osborne, T.F., McAllister, J.M and Strauss, J.F. III. 2001. Conditional response of the human steroidogenic acute regulatory protein gene promoter to sterol regulatory element binding protein1a. Endocrinology 142:28-36.

65. Wang, X., Walsh, L.P., Reinhart, A.J., and Stocco, D.M. 2000. The role of arachidonic acid in steroidogenesis and steroidogenic acute regulatory (StAR) gene and protein expression. J. Biol. Chem. 275:20204-20209.

66. Horton, J.D., Goldstein, J.L., and Brown, M.S. 2002. SREBPs: activators of the complete program of cholesterol and fatty acid synthesis in the liver. J. Clin. Invest. 109:1125-1131. doi:10.1172/JCI200215593.

67. Brand, C., Nury, D., Chambaz, E.M., Feige, J.J., and Bailly, S. 2000. Transcriptional regulation of the gene encoding the StAR protein in the human adrenocortical cell line, H295R, by cAMP and TGFbeta1. Endocr. Res. 26:1045-1053.

68. Manna, P.R., et al. 2001. Assessment of mechanisms of thyroid hormone action in mouse Leydig cells: regulation of the steroidogenic acute regulatory protein, steroidogenesis, and luteinizing hormone receptor function. Endocrinology. 142:319-331.

69. Hales, K.H., et al. 2000. Diametric effects of bacterial endotoxin lipopolysaccharide on adrenal and Leydig cell steroidogenic acute regulatory protein. Endocrinology. 141:4000-4012.

70. Le, T., and Schimmer, B.P. 2001. The regulation of MAPKs in Y1 mouse adrenocortical tumor cells. Endocrinology. 142:4282-4287. 